\title{
Article \\ Performance Evaluation of a THz Pulsed Imaging System: Point Spread Function, Broadband THz Beam Visualization and Image Reconstruction
}

\author{
Marta Di Fabrizio 1,*(D), Annalisa D'Arco ${ }^{2, *}$, , Sen Mou ${ }^{2}$, Luigi Palumbo ${ }^{3}$, Massimo Petrarca ${ }^{2,3}$ and \\ Stefano Lupi ${ }^{1,4}$ \\ 1 Physics Department, University of Rome 'La Sapienza', P.le Aldo Moro 5, 00185 Rome, Italy; \\ stefano.lupi@roma1.infn.it \\ 2 INFN-Section of Rome ‘La Sapienza', P.le Aldo Moro 2, 00185 Rome, Italy; sen.mou@roma1.infn.it (S.M.); \\ massimo.petrarca@uniroma1.it (M.P.) \\ 3 SBAI-Department of Basic and Applied Sciences for Engineering, Physics, University of Rome 'La Sapienza', \\ Via Scarpa 16, 00161 Rome, Italy; luigi.palumbo@uniroma1.it \\ 4 INFN-LNF, Via E. Fermi 40, 00044 Frascati, Italy \\ * Correspondence: martadifabrizio@gmail.com (M.D.F.); annalisa.darco@roma1.infn.it (A.D.)
}

\section{check for} updates

Citation: Di Fabrizio, M.; D'Arco, A.; Mou, S.; Palumbo, L.; Petrarca, M.; Lupi, S. Performance Evaluation of a THz Pulsed Imaging System: Point Spread Function, Broadband THz Beam Visualization and Image Reconstruction. Appl. Sci. 2021, 11, 562. https://doi.org/10.3390/ app11020562

Received: 18 December 2020 Accepted: 5 January 2021 Published: 8 January 2021

Publisher's Note: MDPI stays neutral with regard to jurisdictional clai$\mathrm{ms}$ in published maps and institutional affiliations.

Copyright: (C) 2021 by the authors. Licensee MDPI, Basel, Switzerland. This article is an open access article distributed under the terms and conditions of the Creative Commons Attribution (CC BY) license (https:// creativecommons.org/licenses/by/ $4.0 /)$.

\begin{abstract}
Terahertz (THz) technology is a promising research field for various applications in basic science and technology. In particular, THz imaging is a new field in imaging science, where theories, mathematical models and techniques for describing and assessing $\mathrm{THz}$ images have not completely matured yet. In this work, we investigate the performances of a broadband pulsed $\mathrm{THz}$ imaging system (0.2-2.5 THz). We characterize our broadband $\mathrm{THz}$ beam, emitted from a photoconductive antenna (PCA), and estimate its point spread function (PSF) and the corresponding spatial resolution. We provide the first, to our knowledge, 3D beam profile of $\mathrm{THz}$ radiation emitted from a PCA, along its propagation axis, without the using of $\mathrm{THz}$ cameras or profilers, showing the beam spatial intensity distribution. Finally, we evaluate the THz image formation on a test-sample composed by a regular linen natural pattern.
\end{abstract}

Keywords: $\mathrm{THz}$ radiation; $\mathrm{THz}$ pulsed imaging; point spread function; spatial resolution; $\mathrm{THz}$ 3D beam

\section{Introduction}

In recent years, the terahertz $(\mathrm{THz})$ range of the electromagnetic spectrum $(0.1-10 \mathrm{THz})$ has become very attractive, due to its applications in basic science and technology [1-6]. THz photons ( $1 \mathrm{THz}$ is equivalent to $4 \mathrm{meV}, 300 \mu \mathrm{m}$ and $33 \mathrm{~cm}^{-1}$ ) due to their non-ionizing nature, can be exploited for many nondestructive applications in biomedicine, metrology and quality control of industrial productions, spectroscopic investigations, environmental monitoring, art conservation, particle-accelerator physics [7-15], etc. In addition, $\mathrm{THz}$ waves can propagate through inflammable liquids without causing combustion, resulting in their being suitable for security and gas sensing purposes [16-19]. Moreover, many materials exhibit their spectral fingerprints in this frequency range. THz radiation is very sensitive to polar molecules like water whilst remaining rather transparent to non-polar molecules like plastics, ceramics, paper, cloths, fats, etc. These distinct optical responses are often the basis for both materials' characterization [20] and spectroscopic imaging [7].

In particular, THz time-domain spectroscopy (THz-TDS) is based on the synchronous and coherent detection of a THz pulse: both its amplitude and phase are recorded as a function of time, allowing the measurement of sample optical properties, such as the refractive index and the absorption coefficient, without the need of Kramers-Kronig relations.

Actually, $\mathrm{THz}$ radiation can be produced and detected from a variety of designed $\mathrm{THz}$ emitters and receivers, both in continuous wave (CW) and in pulsed regime. Among 
$\mathrm{THz}$ pulsed radiation, some schemes use photoconductive antennas (PCAs) both for $\mathrm{THz}$ emission and detection [21,22], some others exploit $\mathrm{THz}$ production using inorganic and organic crystals [23,24], or by plasma in air [25] and electro-optic sampling (EOS) [26] for detection, etc.

Despite a vast variety of applications, $\mathrm{THz}$ imaging is a challenging research field. $\mathrm{THz}$ imaging has the ability to look into and through transparent objects such as many material packages like PET, for food inspection; or can be used for tumor margins delineation both on in-vivo and ex-vivo biological tissues [7].

As $\mathrm{THz}$ imaging is rather new field, theories and mathematical models [27] for assessing THz imaging systems [28] are not completely matured yet. Ahi and coauthors [27,29,30] mathematically modeled transmission point spread function (PSF) for wideband THz imaging systems, using it to simulate and enhance the resolution of the THz imaging systems.

Some efforts have been made to characterize the THz beam emitted from PCA [31], crystals [32] and plasma in air [33,34], especially using $\mathrm{THz}$ cameras and microbolometric arrays. Conversely, 3D beam profile of $\mathrm{THz}$ radiation emitted from a PCA, along its propagation axis, without the using of $\mathrm{THz}$ cameras or profilers is lacking. As all imaging systems, also THz-based ones are limited in their performances by various factors, such as spatial resolution, contrast and noise. They, in turn, are affected by THz source, system configuration, measurements setting and data acquisition scheme. In particular, $\mathrm{THz}$ imaging poses a challenge to the spatial resolution that can be achieved. This is strictly related to the diffraction limit and to the PSF dependence on the wavelength [35].

Despite some algorithm and optics-based procedures for enhancing the resolution being proposed, the image quality assessment is necessary for the comparison of different imaging systems, particularly for emerging THz ones [36-40].

This paper presents a study of the performances of our home-made $\mathrm{THz}$ pulsed imaging (TPI) system, in terms of PSF, spatial resolution and image formation. In addition, the 3D broadband $\mathrm{THz}$ beam spatial distribution, emitted from a PCA, is characterized and visualized. In the first section of the paper, we discuss our home-made imaging system, based on a THz-TDS scheme. Then, we discuss the TPI basic principles, evaluating the spatial resolution of our imaging system and providing the first, to our knowledge, 3D image of $\mathrm{THz}$ beam emitted from a PCA, along its propagation axis. Finally, we provide a spatial resolution test using a periodic linen pattern.

\section{Experimental Setup}

Our home-made TPI system is based on a pump-probe configuration in transmission mode that produces broadband $\mathrm{THz}$ radiation in the range $0.2-3 \mathrm{THz}$. The experimental layout is illustrated in Figure 1.

Two twin PCAs (G10620-11, Hamamatsu) are used for both THz generation and detection. The system is powered by a mode-locked femtosecond laser (FemtoFiberNIRpro, Toptica) at $780 \mathrm{~nm}$, with $100 \mathrm{fs}$ pulse duration and $80 \mathrm{MHz}$ repetition rate. A 50:50 beamsplitter splits the laser beam into two parts: the pump and the probe beams (each $15 \mathrm{~mW}$ in power). Dielectric mirrors (M, BB1-E03, Thorlabs) convey the two laser beams towards emitter and receiver PCAs. The pump beam is modulated by the THz emitter bias voltage $(-7 / 7 \mathrm{~V}, 1 \mathrm{kHz})$ and then focused on the THz PCA emitter. The generated $\mathrm{THz}$ radiation is then collimated and focused onto a target-sample by using two polymethylpentene plano-convex lenses (TPX, MenloSystems, $\mathrm{f}=50 \mathrm{~mm}$ ). After the sample, the transmitted $\mathrm{THz}$ beam is recollimated and refocused onto the THz PCA detector using two TPX lenses ( $\mathrm{f}=50 \mathrm{~mm}$ ). Simultaneously, the probe beam is used to gate the THz PCA detector. A motorized delay line (DL, DDSM100/M Thorlabs) is used to temporally and coherently sample the THz electric field, and thus both the amplitude and phase are measured (See Section S1 in Supplementary Materials). The signal from PCA detector is filtered and detected by a lock-in amplifier (LIA, SR530, Stanford). The analog output of LIA is collected and digitalized by a dedicated National Instrument acquisition card at 16-bit (DAQ, NI, 6361-BNC connector) and then transferred to a computer for data collection, 
visualization and off-line analysis. In order to collect $\mathrm{THz}$ images, the sample is aligned perpendicularly to the propagating $\mathrm{THz}$ radiation and can be moved in the $\mathrm{THz}$ beam, by a 3D imaging module. It is formed of a motorized 3D linear translation stage (PT1/M, Thorlabs with $25 \mathrm{~mm}$ travel range and $10 \mu \mathrm{m}$ accuracy and servo actuators Z825B, Thorlabs with $<5 \mu \mathrm{m}$ repeatability) that moves the specimen along the $\mathrm{THz}$ beam propagation axis (z-axis, 1D motion) and perpendicularly to it (x,y-axes, 2D motion). The object surface is sampled on a discrete grid composed of square pixels and an acquisition for each pixel is made. Therefore, the measurement is performed with a step-by-step sampling and it can be acquired in fixed DL or spectral modes. For fixed DL images, the DL is set to a fixed position, for example at the location that corresponds to the maximum of the $\mathrm{THz}$ electric field $\mathrm{E}_{\mathrm{MAX}}$. Thus, only $\mathrm{E}_{\mathrm{MAX}}$ is acquired in each pixel. For spectral images, instead, the information of a complete DL temporal scan is recorded, in each pixel. It allows the reconstruction of the whole THz pulse. By means of Fast Fourier Transform (FFT), images at given frequencies can be extracted from spectral images.

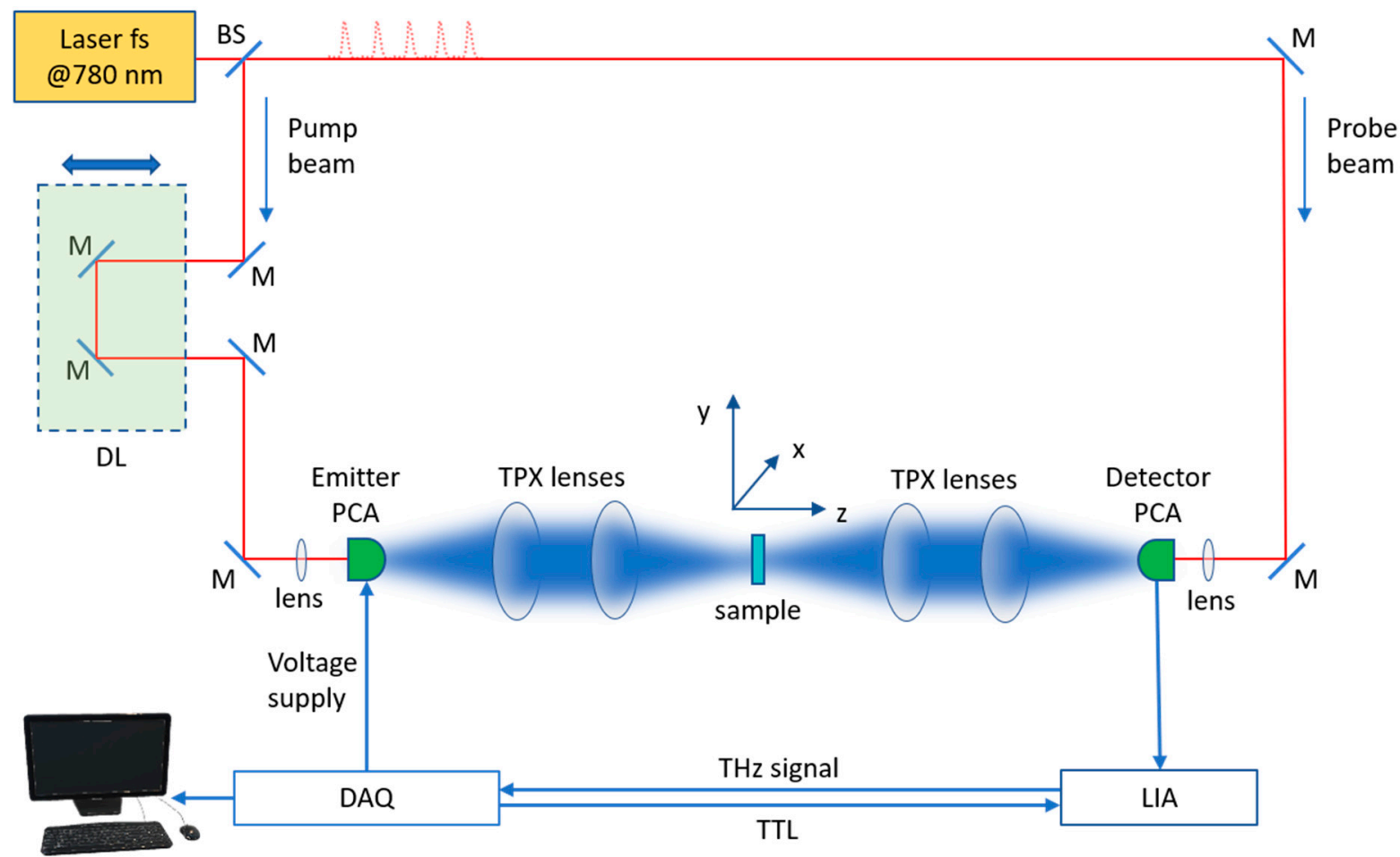

Figure 1. Schematic layout of our THz-TDS setup in transmission mode. Its components are a femtosecond (fs) laser, a beam-splitter (BS), dielectric mirrors (M), a delay line (DL), lenses, THz emitter and detector PCAs, a lock-in amplifier (LIA), a data acquisition card (DAQ) and a computer. See text for details.

\section{Theory}

In this section, we briefly recall the basic concepts of spatial resolution and point spread function (PSF), and later, we report the main quantities for a Gaussian beam. These calculations will be the fundamentals to understand the experimental results found in our work.

\subsection{Spatial Resolution}

The theoretical spatial resolution $R_{T}$ at a certain frequency $(f)$ or wavelength $(\lambda)$ is generally limited by the numerical aperture $(N A)$ of the imaging system and can be defined by the Rayleigh limit [41]

$$
R_{T}=0.61 \frac{\lambda}{N A}=0.61 \frac{c}{N A f}
$$


where $\lambda=c / f$ is the wavelength and $c$ the speed of light in vacuum. As a consequence, $R_{T}(f)$ is strongly frequency-dependent and small objects or any spatial features are easier resolvable at high frequencies. Therefore, in a broadband THz imaging system it is crucial to calculate the spatial resolution for each frequency of interest.

\subsection{Point Spread Function}

PSF is defined as the imaging system response to an ideal, point-like source and constitutes the basic unit forming the image $[27,28,42,43]$ Thus, it represents the optical system's fidelity in reproducing objects through their images. In a perfect imaging system, the intensity coming from a point source (in the object plane) would be concentrated at a single point (in the image plane), the ideal image point. In real systems, however, optical imperfections result in an intensity distribution broadening around the ideal image point, producing blurred images. The PSF provides an estimation of this blurring effect $[42,43]$.

Considering a linear system, the output formed by a sum of inputs is equal to the sum of the outputs corresponding to each input acting separately. Mathematically, if an object $o(x, y)$ is imaged with an imaging system having a response $\operatorname{PSF}(x, y)$, the observed image $i(x, y)$ is given by:

$$
i(x, y)=o(x, y) * \operatorname{PSF}(x, y)
$$

where $x$ and $y$ are plane coordinates of the imaging system, perpendicular to the propagation beam axis and $*$ denotes convolution operation [27]. As $i(x, y)$ is the acquired image, Equation (2) can be inversely used in order to calculate $o(x, y)$, knowing the system $\operatorname{PSF}(x, y)$. PSF of the imaging system is therefore used for improving image quality. Moreover, in a typical TPI system, the THz pulse is broadband, and thus it cannot be treated as a monochromatic beam. Therefore, the PSF is a frequency-dependent quantity: for including the full spectrum, the PSF is reconstructed by superposition of monochromatic beams in the frequency band.

PSF can be evaluated by imaging objects such as tiny reflecting balls [28] and subwavelength holes for reflection and transmission setup geometries, respectively.

\subsection{Gaussian Beam}

Assuming that the $\mathrm{THz}$ beam emitted from a PCA is a Gaussian beam (and this is a very good approximation of actual beam) [27,44,45], it can be modeled as follows $[27,29,43-47]$.

According to Figure 2, the Gaussian beam parameters can be defined and linked to each other. $W_{0}$ is the beam waist size at the focal plane $(z=0)$. The beam waist $W(z)$, along the $z$ direction, enlarges and can be expressed by the following equation:

$$
W(z)=W_{0} \sqrt{1+\left(\frac{z}{Z_{R}}\right)^{2}}
$$

where $Z_{R}$ is called Rayleigh distance and represents the distance along the $z$ axis where $W\left(Z_{R}\right)=\sqrt{2 W_{0}}$. It is defined as follows

$$
Z_{R}=\frac{W_{0}^{2} \pi f}{c}
$$

where $f$ is the frequency and $c$ the speed of light in vacuum. Note that, as shown in Figure 2, the waist sizes, both in the focal region $\left(W_{0}\right)$ and outside $(W(z))$, refer to the Gaussian width $r$ associated to an intensity reduction of a factor $1 / e^{2}$. Moreover, the beam divergence angle $\theta$ can be defined as the ratio between $W_{0}$ and $Z_{R}$ :

$$
\theta=\frac{W_{0}}{Z_{R}}
$$




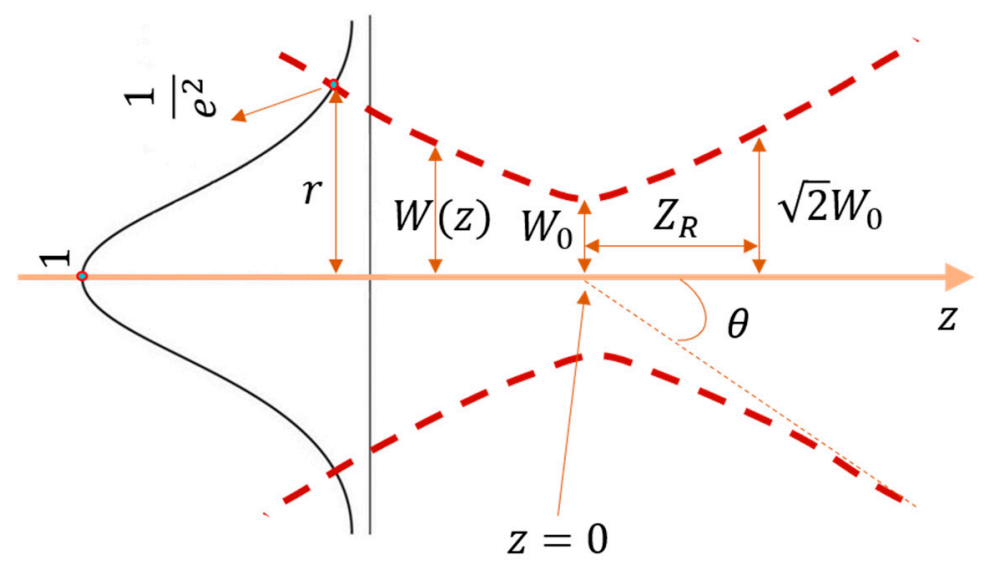

Figure 2. Schematic visualization of Gaussian beam parameters: the beam waist in the focal region $\left(W_{0}\right)$ and along the $z$ propagation axis $(W(z))$, the Rayleigh distance $\left(Z_{R}\right)$, the beam divergence angle $(\theta)$. See text for details.

\section{Results}

\subsection{PSF and Spatial Resolution}

As briefly recalled in Section 3.2, the PSF of an imaging system can be evaluated from sub-wavelength pinhole images $[27,28,42,48]$. We used a $200 \pm 6 \mu \mathrm{m}$ diameter metallic pinhole to simulate a point-like source. We put it in the focal region of our beam $(z=0$, with respect to Figure 2). Note that TPX lenses used for focusing $\mathrm{THz}$ radiation have constant refractive index in our spectral range $(n=1.456 \pm 0.001)$ [49]. Therefore, chromatic aberrations are negligible in our setup. Figure 3 a shows four spectral images at 0.6, 1.0, 1.6 and $2.5 \mathrm{THz}$.

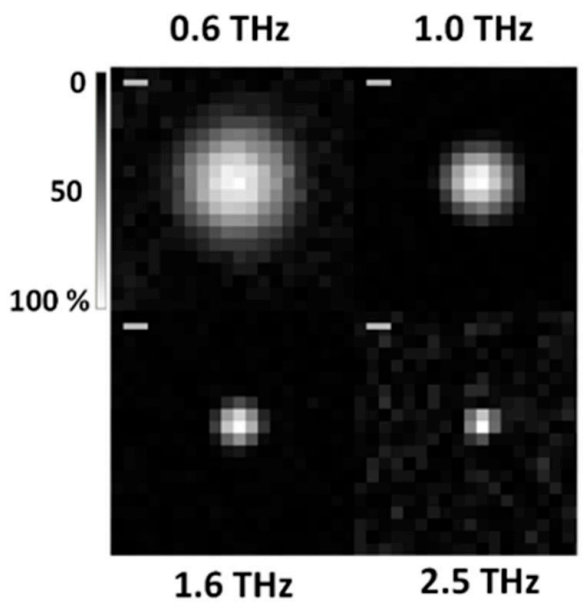

(a)

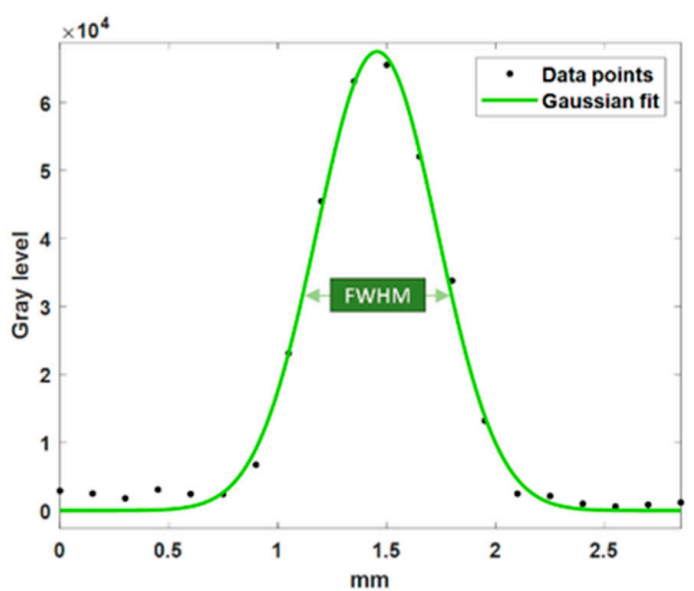

(b)

Figure 3. (a) Four spectral images of the same $200 \mu \mathrm{m}$ pinhole for evaluating our imaging system point spread function (PSF). Figures (20 px $\times 20 \mathrm{px}, 3 \mathrm{~mm} \times 3 \mathrm{~mm})$ are collected at four different frequencies: $0.6,1.0,1.6$ and $2.5 \mathrm{THz}$. The scale bar and the spectral resolution in each image are $0.3 \mathrm{~mm}$ and $0.1 \mathrm{THz}$, respectively. (b) Full Width at Half Maximum (FWHM) estimation from spot intensity profile. Black: experimental data. Green: Gaussian fitting curve. Data shown for $1.0 \mathrm{THz}$ image.

Point-like source is seen very differently depending on what frequency is selected. In order to evaluate the PSF, the spot intensity profile is plotted as a function of distance (black points in Figure 3b, data shown for $1.0 \mathrm{THz}$ image). The Full Width at Half Maximum (FWHM) has been calculated from the Gaussian fit for each image in Figure 3a. With 
this method, we estimated how the point-like source is seen from the imaging system. This coincides with the theoretical spatial resolution at a given frequency estimated for our imaging system. Figure $3 \mathrm{~b}$ reports the experimental data (black) and the Gaussian fit (green) performed to extract the FWHM, at $1.0 \mathrm{THz}$. Gray level refers to the digital information contained in each pixel. It is a discrete value between 0 and 65565, because images have been acquired with a 16-bit data acquisition card (see Section 2 and Section S2 in Supplementary Materials).

Note that the Gaussian fit has been performed using the Gaussian function $f(x)$

$$
f(x)=\frac{1}{\sigma \sqrt{2 \pi}} e^{-\frac{(x-\mu)^{2}}{2 \sigma^{2}}}
$$

where $\mu$ and $\sigma$ are the Gaussian expected value and variance, respectively used as fit parameters. According to [47], the FWHM is:

$$
F W H M=2 \sqrt{2 \ln 2} \sigma
$$

The Table 1 below lists the FWHMs extracted from Figure 3a at various frequencies.

Table 1. FWHMs calculated by the Gaussian fit, at different frequencies, following Equation (7).

\begin{tabular}{cc}
\hline Frequency (THz) & FWHM (mm) \\
\hline 0.6 & $1.06 \pm 0.02$ \\
1.0 & $0.67 \pm 0.01$ \\
1.6 & $0.42 \pm 0.01$ \\
2.5 & $0.29 \pm 0.02$ \\
\hline
\end{tabular}

As can be seen in Table 1, the FWHM decreases as the frequency increases. We adopted the procedure explained above for all the images at fixed frequencies in the range 0.5-2.5 THz. In Figure 4, we report the extracted FWHM, i.e., the spatial resolution, at different frequencies (black points) and the green curve fitting (Equation (1)).

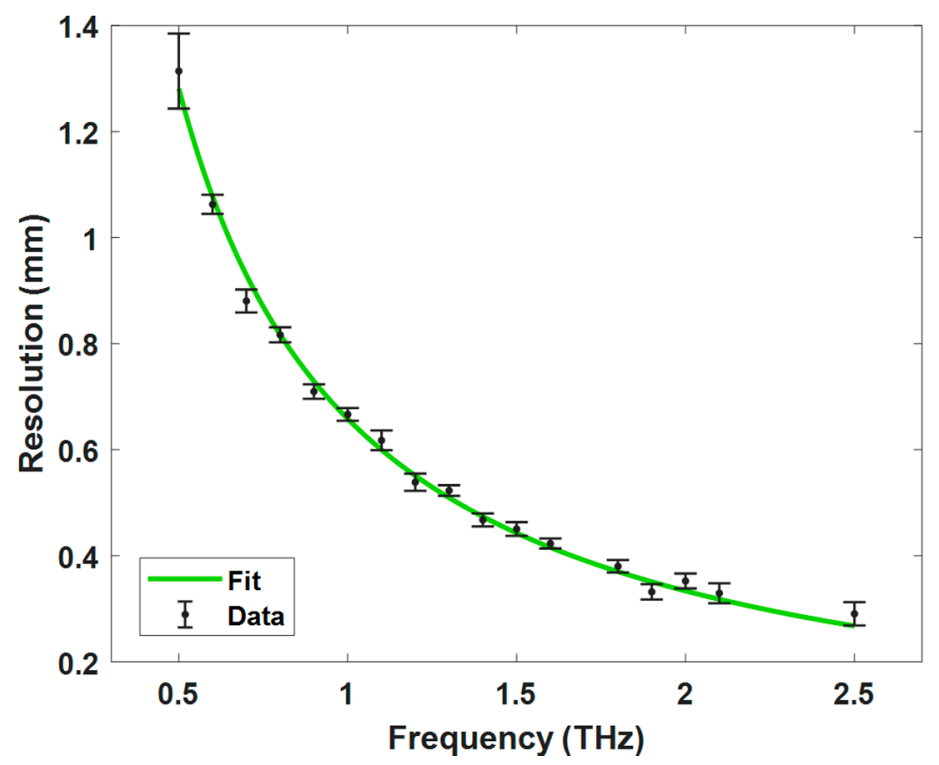

Figure 4. Spatial resolution vs frequency. In black: Full Width at Half Maximum (FWHM) calculated according to Equations (6) and (7), at different frequencies. Black: experimental data. Green: our imaging system spatial resolution curve. It was obtained by fitting the data with the Rayleigh limit Equation (1). 
As expected, the measured resolution data points show a hyperbolic trend as the frequency increases and it is in total agreement with Equation (1). The spatial resolution is higher as the frequency increases. Note that we selected the frequency range $0.5-2.5 \mathrm{THz}$ because for low frequencies $(f<0.5 \mathrm{THz}$ ) the diffractive effects caused by the pinhole were too strong and no luminous spot was clearly identifiable; for high frequencies ( $\mathrm{f}>2.5 \mathrm{THz}$ ) the signal-to-noise ratio (SNR) $[50,51]$ was too low and no detectable intensity was clearly measurable.

By fitting the data with Equation (1), the NA experimental value for our broadband imaging system has been extracted and estimated $(\mathrm{NA}=0.27 \pm 0.01)$.

\section{2. $\mathrm{THz} 3 \mathrm{D}$ Beam Image}

In order to realize a $3 \mathrm{D}$ visualization of $\mathrm{THz}$ beam emitted from the PCA, we spatially selected the beam and acquired fixed DL images at different $z$ positions. We used a $1.20 \pm$ $0.05 \mathrm{~mm}$ diameter metallic pinhole, moved along $x, y$ and $z$ directions. Each image (60 px $\times 60 \mathrm{px}, 15 \mathrm{~mm} \times 15 \mathrm{~mm})$ was acquired in-plane $(x, y)$ at fixed $z$ positions, collecting a $z$ stack-image. The $z$ step was set to $1 \mathrm{~mm}$. The spot size for each image was extracted by a Gaussian fit. According to the following equation, the spot radius $r_{S}$ is defined as

$$
r_{s}=2 \sigma
$$

In Figure 5, we report the procedure for the image located at $z=-3 \mathrm{~mm}$ ( $3 \mathrm{~mm}$ before the $\mathrm{THz}$ beam focal plane), as an example.

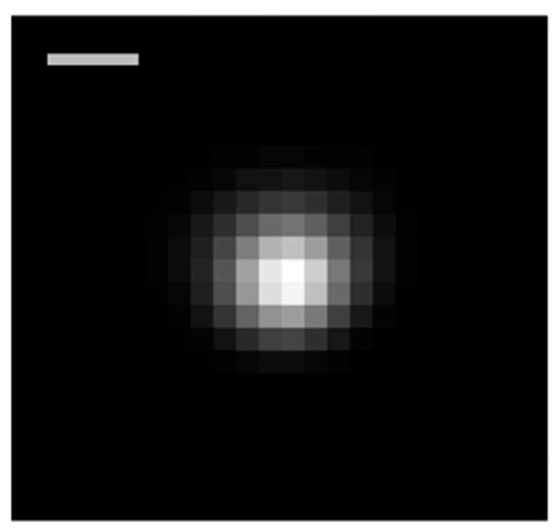

(a)

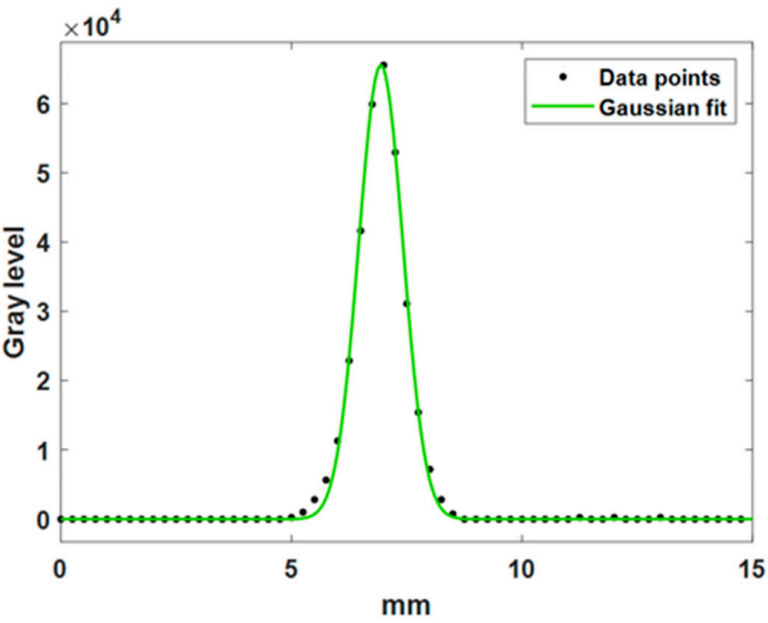

(b)

Figure 5. (a) Crop of 2D acquired image ( $60 \mathrm{px} \times 60 \mathrm{px}, 15 \mathrm{~mm} \times 15 \mathrm{~mm})$ of THz beam at $z=-3 \mathrm{~mm}$ ( $3 \mathrm{~mm}$ before the focal point). (b) Intensity plot as a function of distance. The scale bar is $1 \mathrm{~mm}$.

Figure 5a represents a crop of the acquired image at $z=-3 \mathrm{~mm}$ in grey look-up table (LUT). Figure $5 \mathrm{~b}$ shows its intensity profile as a function of distance (black dots) and the Gaussian fit (green curve). The 3D Volume Viewer tool by ImageJ was used to convert the $z$-stack image into a 3D volume, in Figure 6.

As expected, the maximum intensity locates where the $\mathrm{THz}$ beam has its smallest diameter, i.e., the focal region. Figure 6 is formed of a stack of fixed DL images, therefore no information about $\mathrm{THz}$ frequencies spatial distribution is known. The 3D image in Figure 6 is a result of all frequency contributions, each one with its own weight (See Figure S2 in Supplementary Materials for THz power spectrum vs. frequency).

The knife edge method was adopted for reconstructing the $1 \mathrm{D} \mathrm{THz}$ beam profile $[46,52,53]$ and for comparing the results with the pinhole method. A sharp metallic edge was placed vertically within the $\mathrm{THz}$ beam and was moved along $x$ and $z$ directions (the edge position along $y$ was kept fixed for all the knife edge measurements). Each fixed 
$D L$ image was acquired by varying $x$ position, at different $z$-fixed positions. The $z$ step was set to $1 \mathrm{~mm}$.

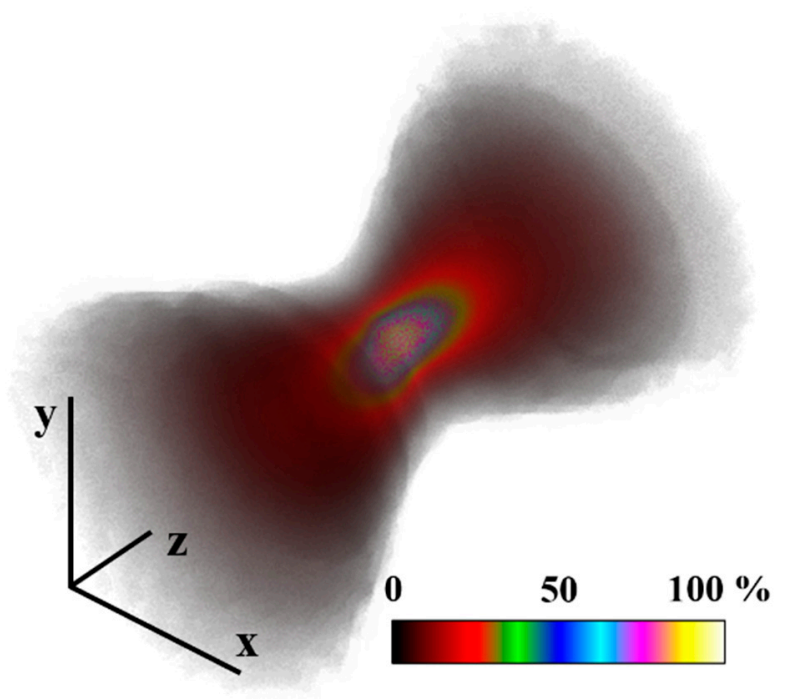

Figure 6. 3D image of our $\mathrm{THz}$ beam emitted from PCA. The percentual scale is referred to the intensity of the $\mathrm{THz}$ beam. The image shows a $\mathrm{THz}$ beam double-cone shape, where the higher intensity values are in its focal region. Scale bars: $2.5 \mathrm{~mm}$ along $x$ and $y, 5 \mathrm{~mm}$ along $z$.

In general, a sharp edge moving in a light beam having electric field $E$, can be modeled by a function containing the error function $\operatorname{erf}(x)$ [52]:

$$
E(x, z)=\frac{E_{M A X}}{2}\left[1+\operatorname{erf}\left(\frac{\sqrt{2}\left(x-x_{50 \%}\right)}{W(z)}\right)\right]
$$

where $E(x, z)$ is the THz electric field as a function of distances along $x$ and $z$ directions, $E_{M A X}$ is the maximum $\mathrm{THz}$ electric field, $x_{50 \%}$ the position along $x$ corresponding to $E_{M A X} / 2$ and $W(z)$ is the THz beam waist as a function of distance $z$, along the propagation axis. This equation weights all the frequency contributions because no frequency is selected in fixed DL images. By fitting the data points with Equation (9), the green curve is obtained and displayed in Figure 7.

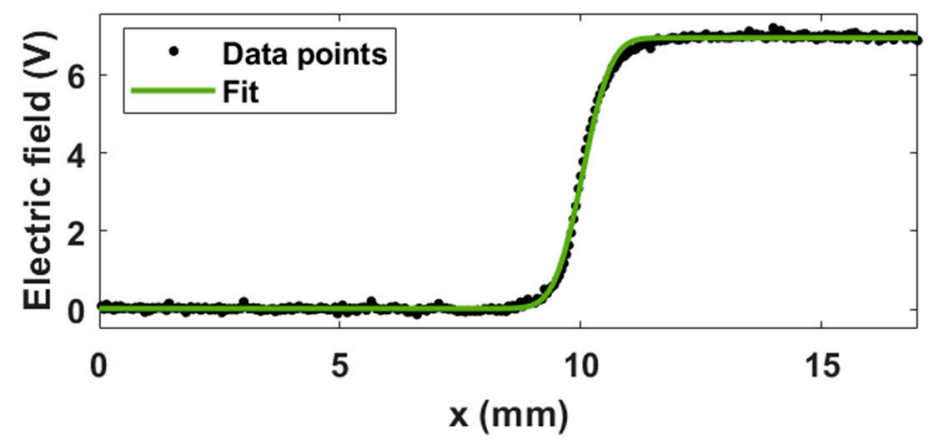

Figure 7. Electric field profile with knife edge method at $z=-3 \mathrm{~mm}$. Black: electric field data points as a function of distance along the $x$ direction (perpendicular to the beam propagation $z$ axis). Green: fitting curve using Equation (9).

This was performed for each image at each $z$ position. The beam waist size $W(z)$, at different $z$ positions, have been extracted from the fitting parameters, using Equation (9). Figure 8 shows the results for the two methods adopted for the beam profile reconstruction. 


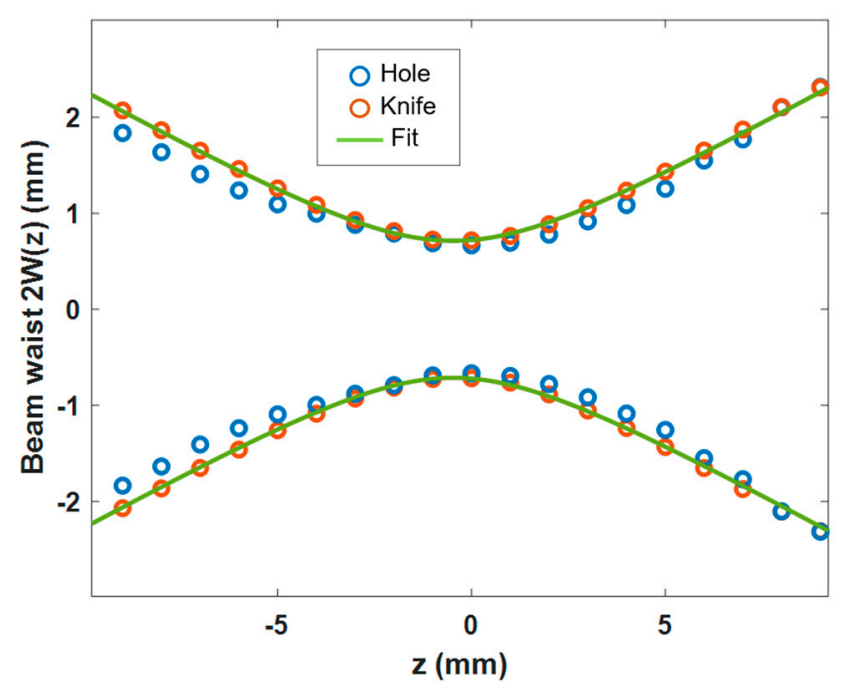

Figure 8. Comparison between the two methods adopted for $\mathrm{THz}$ beam profiling (hole and knife edge methods) and curve fit. In blue: hole, in orange: knife, in green: fitting curve using Equation (3).

In particular, the quantity $2 W(z)$ is plotted in Figure 8, and therefore the $\mathrm{THz}$ beam 1D projection is shown $1.2 \mathrm{~cm}$ before and after the focal region. The blue circles represent the pinhole method, the oranges show the knife edge method: they are well in agreement. By fitting the data points with Equation (3), the relevant parameters for a Gaussian beam, defined in Section 3.3, have been found. They are listed in Table 2.

Table 2. Our THz beam relevant quantities: waist at the focal point $\left(W_{0}\right)$, Rayleigh distance $\left(Z_{R}\right)$ and divergence angle $(\theta)$.

\begin{tabular}{ccc}
\hline$W_{\mathbf{0}}(\mathbf{m m})$ & $\boldsymbol{Z}_{\boldsymbol{R}}(\mathbf{m m})$ & $\boldsymbol{\theta}\left(^{\circ}\right)$ \\
\hline $0.69 \pm 0.02$ & $3.1 \pm 0.1$ & $12 \pm 1$ \\
\hline
\end{tabular}

\subsection{Sample Test: Linen}

After studying the spatial distribution properties and the capability to resolve objects of our TPI system, we used this experimental apparatus on a sample test: a natural linen pattern. This is a quite regular pattern with horizontal and vertical linen fibers, separated by air holes (see Figure 9f). Each vertical fiber width is $\sim 0.40 \pm 0.05 \mathrm{~mm}(\sim 20$ fibers $/ \mathrm{cm})$ and each horizontal fiber $\sim 0.15 \pm 0.05 \mathrm{~mm}(\sim 17$ fibers $/ \mathrm{cm})$. Holes sizes vary from $\sim 50 \mu \mathrm{m}$ to $\sim 600 \mu \mathrm{m}$.

Linen spectral images have been acquired. We collected transmittance images at different frequencies. Each pixel contains the transmittance value at selected $\mathrm{THz}$ frequencies. The results have been reported in Figure 9a-e, at frequencies $0.2,0.7,0.8,1.0$ and $1.3 \mathrm{THz}$, thanks to the absence of sample absorption spectral features in this spectral range (see Section S5 in Supplementary Materials).

The transmittance without gain $\left(T_{\text {real }}\right)$ for each pixel in each image, can be obtained by using the following Equation (10):

$$
T_{\text {real }}=\frac{T_{\text {displayed }}}{g}
$$

where $T_{\text {displayed }}$ is the transmittance for each pixel, displayed in Figure 9a-e, after applying a gain $g$. The value of $g$, for each image, was chosen in such a way that the maximum transmittance value becomes $100 \%$ transmittance. By increasing the frequency, the spatial features are better discriminated. $g$ is reported under each Figure 9a-e. 


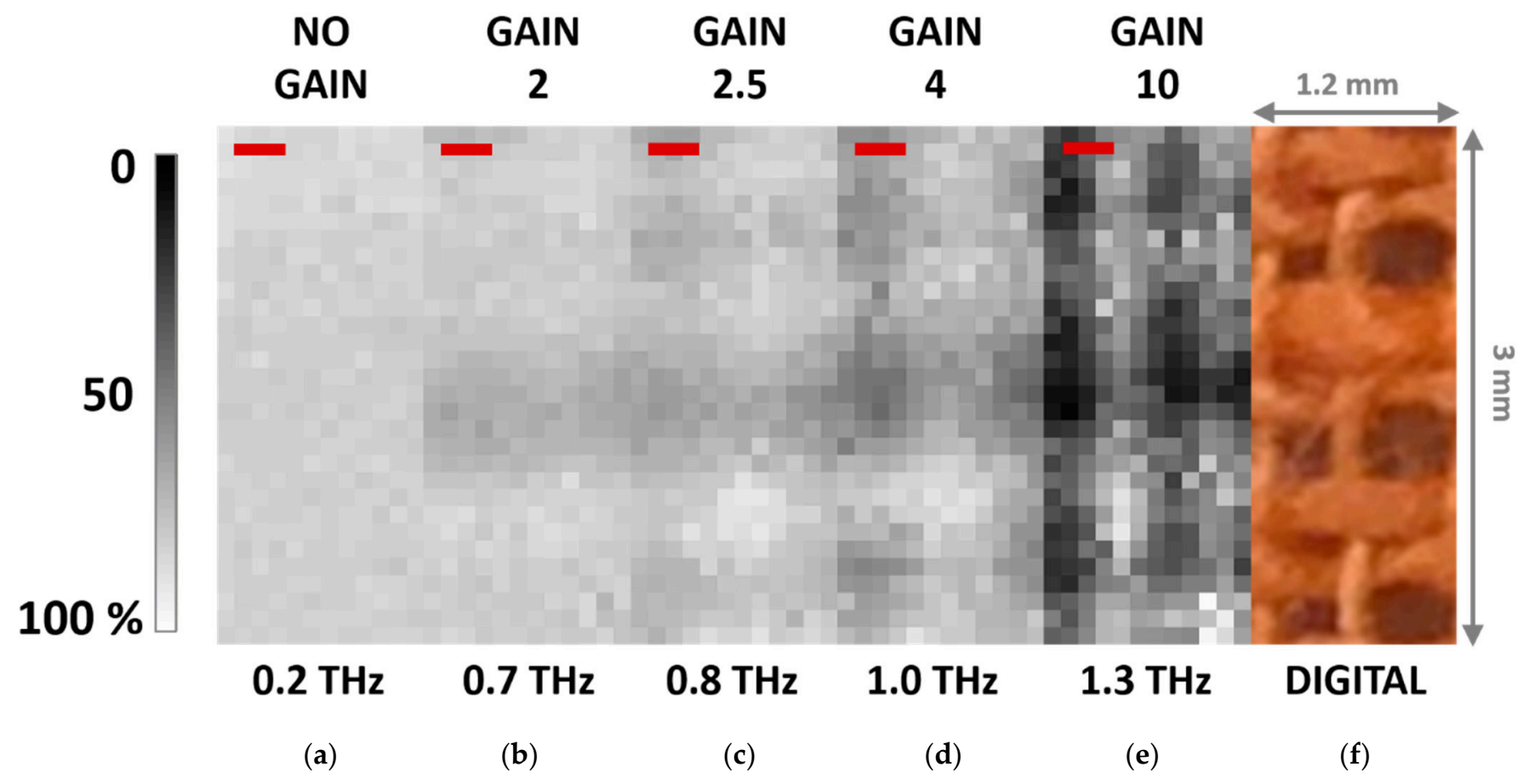

Figure 9. Six images of the same linen sample. (a-e) Five spectral images $(12 \mathrm{px} \times 30 \mathrm{px}, 1.2 \mathrm{~mm} \times 3 \mathrm{~mm})$ at $0.2,0.7,0.8,1.0$, $1.3 \mathrm{THz}$. They have different gain $g$ values, see Equation (10). The scale bar is $0.3 \mathrm{~mm}$ and the spectral resolution is $0.1 \mathrm{THz}$. $100 \%$ and $0 \%$ transmittance are referred to white and black gray levels, respectively. (f) Digital image.

The image corresponding to $0.2 \mathrm{THz}$ is a uniform distribution of intensity pixel values. The low contrast and spatial resolution result in no distinguishable linen structure, due to its strong sub-diffraction nature. At $0.7 \mathrm{THz}$, a horizontal dark line starts to become visible. It is associated to the presence of a not resolved linen fiber (or more). Moving to high frequencies and increasing the spatial resolution, more linen fibers are identifiable, as it is fibers (black zones) are visible, while the white zones are the hole between linen fibers. Figure $9 \mathrm{f}$ shows the digital image and can be compared to the $1.3 \mathrm{THz}$ image, that has the best resolution, among linen spectral images. The two vertical linen fibers are well resolved because spaced by big holes $(>150 \mu \mathrm{m})$, while the three horizontal pairs are not resolved and only three dark regions are visible.

In order to obtain image de-blurring, we performed image deconvolution. With respect to Equation (2), we chose Figure 10a (1.3 THz linen image) as the object $o(x, y)$ being the most resolved linen image and $10 \mathrm{~b}(200 \mu \mathrm{m}$ pinhole image at $1.3 \mathrm{THz})$ as $\operatorname{PSF}(x, y)$. By deconvolving them with ImageJ deconvolution tool, we obtained Figure 10c.

As seen in the de-blurred Figure 10c, the dark regions, corresponding to the linen fibers, become better identifiable, thus an image quality enhancement is visible, resulting in a sharper image. In Figure 10d,e we report the intensity profile and its derivative, for both the original and deconvolved linen images. The red boxes in Figure 10a,c show the pixel lines selected for intensity profile and derivative plots. In Figure 10e $D_{1}$ and $D_{2}$ denote the differences between derivative maximum and minimum for original and deconvolved images, respectively. By computing the ratio $R=D_{2} / D_{1}$ an estimation of the quality improvement can be given. The deconvoluted image shows a 1.6-fold quality improvement. 


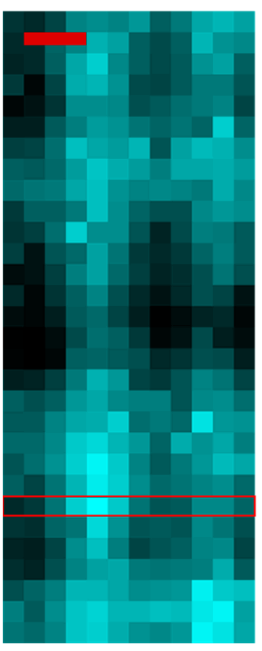

(a)

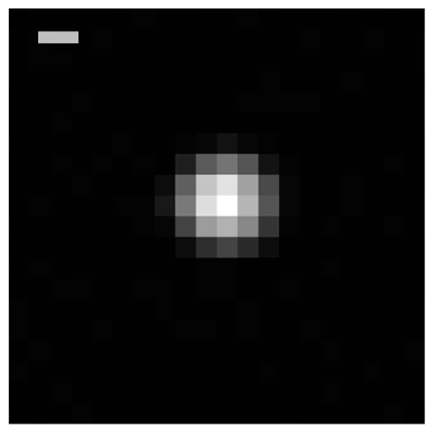

(b)

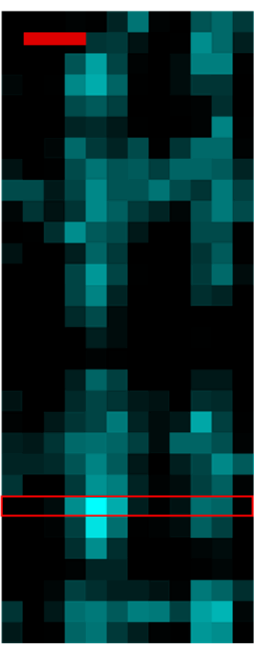

(c)

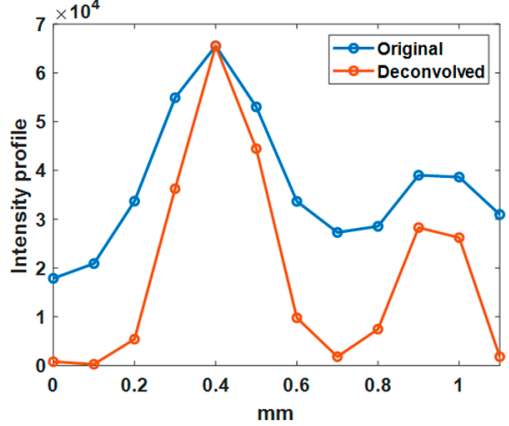

(d)

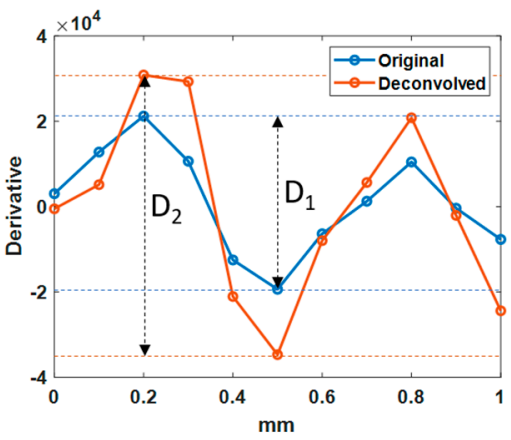

(e)

Figure 10. Images used for deconvolution process. (a) Object image: $1.3 \mathrm{THz}$ linen image, (b) PSF image: $200 \mu \mathrm{m}$ pinhole at $1.3 \mathrm{THz},(\mathbf{c})$ Restored image: deconvolution of (a) and (b). The scale bar is $0.3 \mathrm{~mm}$ for (a) and (c) and $1 \mathrm{~mm}$ for (b). (d) Intensity profile of original (blue) and deconvolved (orange) images. (e) Derivative of original (blue) and deconvolved (orange) images. The selected regions for intensity profiles and derivatives are indicated with red boxes in (a) and (c). $\mathrm{D}_{1}$ and $\mathrm{D}_{2}$ in (e) indicate the differences between derivative maximum and minimum for original and deconvolved images.

\section{Conclusions}

In this paper we characterize a THz-TDS system based on PCAs. We evaluate the performances of the system such as its spectral range emission and spatial resolution. Moreover, we reconstruct the 1D and 3D profiles of our $\mathrm{THz}$ beam, with two different methods: hole and knife edge. Both methods gave results that are in great accordance. Finally, we present a very simple and cheap resolution test that can be done with a linen pattern sample. We demonstrated that our system has the capability to spatially resolve objects with dimensions from $\sim 1.3$ to $\sim 0.3 \mathrm{~mm}$ in the frequency range $0.5-2.5 \mathrm{THz}$, with a resolution of $\sim 0.7 \mathrm{~mm}$ at $1 \mathrm{THz}$. Finally, with the help of the experimental PSF, we show that the $\mathrm{THz}$ image quality can be considerably improved.

Supplementary Materials: The following are available online at https:/ /www.mdpi.com/2076-341 7/11/2/562/s1, Figure S1: THz electric field vs. time, Figure S2: THz power spectrum vs. frequency, Figure S3: THz image background noise at $1.0 \mathrm{THz}$, Figure S4: THz image SNR vs. frequency, Figure S5: THz Linen Transmittance.

Author Contributions: Conceptualization, M.D.F., A.D. and S.L.; formal analysis, M.D.F. and A.D.; writing-original draft preparation, M.D.F., A.D. and S.L.; writing-review and editing, M.D.F., A.D., S.M., L.P., M.P. and S.L.; supervision, S.L.; project administration, S.L.; funding acquisition, S.L. All authors have read and agreed to the published version of the manuscript.

Funding: This research was funded by INFN, in the framework of Commissione V Call THz-ERA (TERA).

Data Availability Statement: The data presented in this study are available in "Performance Evaluation of a THz Pulsed Imaging System: Point Spread Function, Broadband THz Beam Visualization and Image Reconstruction" and in Supplementary Materials. 
Conflicts of Interest: The authors declare no conflict of interest.

\section{References}

1. Tofani, S.; Fuscaldo, W. Fabry-perot cavity leaky wave antennas with tunable features for terahertz applications. Condens. Matt. Phys. 2020, 5, 1-11. [CrossRef]

2. Rezvani, S.J.; Di Gioacchino, D.; Tofani, S.; D’Arco, A.; Ligi, C.; Lupi, S.; Gatti, C.; Cestelli Guidi, M.; Marcelli, A. A cryogenic magneto-optical device for long wavelength radiation. Rev. Sci. Instrum. 2020, 91, 075103. [CrossRef] [PubMed]

3. Pi, H.; Rahman, T.; Boden, S.A.; Ma, T.; Yan, J.; Fang, X. Integrated vortex beam emitter in the THz frequency range: Design and simulation. APL Photonics 2020, 5, 076102. [CrossRef]

4. Delgado-Notario, J.A.; Clericò, V.; Diez, E.; Velázquez-Pérez, J.E.; Taniguchi, T.; Watanabe, K.; Otsuji, T.; Meziani, Y.M. Asymmetric dual-grating gates graphene FET for detection of terahertz radiations. APL Photonics 2020, 5, 066102. [CrossRef]

5. Jazbinšek, M.; Puc, U.; Abina, A.; Zidansek, A. Organic crystals for THz photonics. Appl. Sci. 2019, 9, 882-926. [CrossRef]

6. Guerboukha, H.; Nallappan, K.; Skorobogatiy, M. Toward real-time terahertz imaging. Adv. Opt. Photonics 2018, 10, 843-938. [CrossRef]

7. D'Arco, A.; Di Fabrizio, M.; Dolci, V.; Petrarca, M.; Lupi, S. THz pulsed imaging in biomedical applications. Condens. Matt. Phys. 2020, 5, 1-25. [CrossRef]

8. Rothbart, N.; Holz, O.; Koczulla, R.; Schmalz, K.; Hübers, H.W. Analysis of human breath by millimeter-wave/terahertz spectroscopy. J. Sens. 2019, 19, 2719. [CrossRef]

9. Ren, A.; Zahid, A.; Fan, D.; Yang, X.; Imran, M.A.; Alomainy, A.; Abasi, Q.H. State-of-the-art in terahertz sensing for food and water security-A comprehensive review. Trends Food Sci. Tech. 2019, 85, 241-251. [CrossRef]

10. Wang, K.; Sun, D.W.; Pu, H. Emerging non-destructive terahertz spectroscopic imaging technique: Principle and applications in the agri-food industry. Trends Food Sci. Tech. 2017, 67, 93-105. [CrossRef]

11. Neu, J.; Ostresh, S.; Regan, K.P.; Spies, J.A.; Schmuttenmaer, C.A. Influence of dye sensitizers on charge dynamics in $\mathrm{SnO}_{2}$ nanoparticles probed with THz spectroscopy. J. Phys. Chem. C 2020, 124, 3482-3488. [CrossRef]

12. D'Arco, A.; Mussi, V.; Petrov, S.; Tofani, S.; Petrarca, M.; Beccherelli, R.; Dimitrov, D.; Marinova, V.; Lupi, S.; Zografopoulos, D. Fabrication and spectroscopic characterization of graphene transparent electrodes on flexible cyclo-olefin substrates for terahertz electro-optic applications. Nanotechnology 2020, 31, 364006. [CrossRef] [PubMed]

13. Melinger, J.S.; Yang, Y.; Mandehgar, M.; Grischkowsky, D. THz detection of small molecule vapors in the atmospheric transmission windows. Opt. Expr. 2012, 20, 6788-6807. [CrossRef]

14. Cosentino, A. Terahertz and cultural heritage science: Examination of art and archeology. Technologies 2016, 4, 1-13. [CrossRef]

15. Curcio, A.; Marocchino, A.; Dolci, V.; Lupi, S.; Petrarca, M. Resonant plasma excitation by single-cycle THz pulses. Sci. Rep. 2018, 8, 1-8. [CrossRef] [PubMed]

16. D'Arco, A.; Di Fabrizio, M.; Dolci, V.; Marcelli, A.; Petrarca, M.; Della Ventura, G.; Lupi, S. Characterization of volatile organic compounds (VOCs) in their liquid-phase by terahertz time-domain spectroscopy. Biomed. Opt. Express. 2020, 11, 1-7. [CrossRef]

17. Ergun, S.; Sonmez, S. Terahertz technology for military applications. J. Assoc. Inf. Sci. Technol. 2015, 3, 13-16. [CrossRef]

18. Federici, J.F.; Schulkin, B.; Huang, F.; Gary, D.; Barat, R.; Oliveira, F.; Zimdars, D. THz imaging and sensing for security applications-explosives, weapons and drugs. Semicond. Sci. Technol. 2005, 20, S266. [CrossRef]

19. Mittleman, D.M.; Jacobsen, R.H.; Neelamani, R.; Baraniuk, R.G.; Nuss, M.C. Gas sensing using terahertz time-domain spectroscopy. Appl. Phys. B 1998, 67, 379-390. [CrossRef]

20. Dorney, T.D.; Baraniuk, R.G.; Mittleman, D.M. Material parameter estimation with terahertz time-domain spectroscopy. J. Opt. Soc. Am. A 2001, 18, 1562-1571. [CrossRef]

21. Tani, M.; Herrmann, M.; Sakai, K. Generation and detection of terahertz pulsed radiation with photoconductive antennas and its application to imaging. Meas. Sci. Technol. 2002, 13, 1739. [CrossRef]

22. Burford, N.M.; El-Shenawee, M.O. Review of terahertz photoconductive antenna technology. Opt. Eng. 2017, 56, 010901. [CrossRef]

23. D'Arco, A.; Tomarchio, L.; Dolci, V.; Di Pietro, P.; Perucchi, A.; Mou, S.; Petrarca, M.; Lupi, S. Broadband anisotropic optical properties of the terahertz generator HMQ-TMS organic crystal. Condens. Matt. Phys. 2020, 5, 47-57. [CrossRef]

24. Novelli, F.; Guchhait, B.; Havenith, M. Towards intense THz spectroscopy on water: Characterization of optical rectification by $\mathrm{GaP}, \mathrm{OH} 1$, and DSTMS at OPA wavelengths. Materials 2020, 13, 1311. [CrossRef]

25. Dai, J.; Liu, J.; Zhang, X.C. Terahertz wave air photonics: Terahertz wave generation and detection with laser-induced gas plasma. IEEE J. Sel. Top. Quantum Electron. 2010, 17, 183-190. [CrossRef]

26. Ilyakov, I.E.; Kitaeva, G.K.; Shishkin, B.V.; Akhmedzhanov, R.A. The use of DSTMS crystal for broadband terahertz electro-optic sampling based on laser pulse amplitude changes. Laser Phys. Lett. 2018, 15, 125401. [CrossRef]

27. Ahi, K. Mathematical modeling of THz Point Spread Function and simulation of THz imaging systems. IEEE Trans. Terahertz Sci. Technol. 2017, 7, 747-754. [CrossRef]

28. Popescu, D.C.; Hellicar, A.D. Point spread function estimation for a Terahertz imaging system. EURASIP J Adv. Sig. Process. 2010, 2010, 575817. [CrossRef]

29. Ahi, K.; Anwar, M. Developing terahertz imaging equation and enhancement of the resolution of terahertz images using deconvolution. In Proceedings of the SPIE Commercial + Scientific Sensing and Imaging, Baltimore, MD, USA, 17-21 April 2016 
30. Ahi, K.; Shahbazmohamadi, S.; Asadizanjani, N. Quality control and authentication of packaged integrated circuits using enhanced-spatial resolution terahertz time-domain spectroscopy and imaging. Opt. Lasers Eng. 2018, 104, 274-284. [CrossRef]

31. Oden, J.; Meilhan, J.; Lalanne-Dera, J.; Roux, J.-F.; Garet, F.; Coutaz, J.-L.; Simeons, F. Imaging of broadband terahertz beams using an array of antenna-coupled microbolometers operating at room temperature. Opt. Express 2013, 21, 4817-4825. [CrossRef]

32. Shalaby, M.; Hauri, C.P. Terahertz brightness at the extreme: Demonstration of a $5 \mathrm{GV} / \mathrm{m}, 17 \mathrm{~T}$ low frequency $\lambda 3$ terahertz bullet. arXiv 2014, arXiv:1407.1656.

33. Zhao, J.; Chu, W.; Wang, Z.; Peng, Y.; Gong, C.; Lin, L.; Zhu, Y.; Liu, W.; Cheng, Y.; Zhuang, S.; et al. Strong spatial confinement of terahertz wave inside femtosecond laser filament. ACS Photonics 2016, 3, 2338-2343. [CrossRef]

34. Blank, V.; Thomson, M.D.; Roskos, H.G. Spatio-spectral characteristics of ultra-broadband THz emission from two-colour photoexcited gas plasmas and their impact for nonlinear spectroscopy. New J. Phys. 2013, 15, 075023. [CrossRef]

35. Saleh, B.E.A.; Teich, M.C. Fundamentals of Photonics; John Wiley and Sons: Hoboken, NJ, USA, 2007.

36. Trofimov, V.A.; Trofimov, V.V. New opportunities for quality enhancing of images captured by passive THz camera. In Proceedings of the SPIE Security + Defence 2014, Amsterdam, The Netherlands, 22-25 September 2014.

37. Trofimov, V.A.; Trofimov, V.V. New algorithm for the passive THz image quality enhancement. In Proceedings of the SPIE Defense + Commercial Sensing 2016, Baltimore, MD, USA, 17-21 April 2016.

38. Trofimov, V.A.; Trofimov, V.V.; Kovalev, V.Y.; Loginova, M.M. About efficiency of correlation function using with Fourier transform for the passive THz image quality enhancement. In Proceedings of the SPIE Security + Defence, Warsaw, Poland, 11-14 September 2017.

39. Nguyen Pham, H.H.; Hisatake, S.; Minin, O.V.; Nagatsuma, T.; Minin, I.V. Enhancement of spatial resolution of terahertz imaging systems based on terajet generation by dielectric cube. APL Photonics 2017, 2, 056106. [CrossRef]

40. Chernomyrdin, N.V.; Frolov, M.E.; Lebedev, S.P.; Reshetov, I.V.; Spektor, I.E.; Tolstoguzov, V.L.; Karasik, V.E.; Khorokhorov, A.M.; Koshelev, K.I.; Schadko, A.O.; et al. Wide-aperture aspherical lens for high-resolution terahertz imaging. Rev. Sci. Instrum. 2017, 88, 014703. [CrossRef] [PubMed]

41. Ernst, A. Beitrage zur Theorie des Mikroskops und der mikroskopischen Wahrnehmung. Archiv. Mikrosk. Anat. 1873, 9, 413-468.

42. Rossman, K. Point spread-function, line spread-function, and modulation transfer function: Tools for the study of imaging systems. Radiology 1969, 93, 257-272. [CrossRef]

43. Brown, E.R. Fundamentals of terrestrial millimeter-wave and THz remote sensing. Int. J. High Speed Electron. Syst. 2003, 13, 995-1097. [CrossRef]

44. Marshall, G.F.; Stutz, G.E. Handbook of Optical and Laser Scanning; CRC Press: Boca Raton, FL, USA, 2016.

45. Gürtler, A.; Winnewisser, C.; Helm, H.; Uhd Jepsen, P. Terahertz pulse propagation in the near field and the far field. J. Opt. Soc. Am. A 2000, 17, 74-83. [CrossRef]

46. Magnes, J.; Odera, D.; Hartke, J.; Fountain, M.; Florence, L.; Davis, V. Quantitative and qualitative study of Gaussian beam visualization techniques. arXiv 2006, arXiv:physics/06051.

47. Paschotta, R. Field Guide to Laser Pulse Generation; SPIE Publications: Bellingham, WA, USA, 2008.

48. Gonzalez, R.C.; Woods, R.E. Digital Image Processing; Pearson Prentice Hall: London, UK, 2002.

49. Podzorov, A.; Guilhem, G. Low-loss polymers for terahertz applications. Appl. Opt. 2008, 47, 3254-3257. [CrossRef] [PubMed]

50. Naftaly, M.; Dudley, R. Methodologies for determining the dynamic ranges and signal-to-noise ratios of terahertz time-domain spectrometers. Opt. Lett. 2009, 34, 1213-1215. [CrossRef]

51. Naftaly, M. Terahertz Metrology; Artech House Publishers: Norwood, MA, USA, 2015.

52. Diaz-Uribe, R.; Rosete-Aguilar, M.; Ortega-Martinez, R. Position sensing of a Gaussian beam with a power meter and a knife edge. Rev. Mex. Fis. 1992, 39, 484-492.

53. De Araujo, M.A.; Silva, R.; de Lima, E.; Pereira, D.P.; de Oliveira, P.C. Measurement of Gaussian laser beam radius using the knife-edge technique: Improvement on data analysis. Appl. Opt. 2009, 48, 393-396. [CrossRef] [PubMed] 